\title{
Excitonic Calculations of ELNES: Low Energy and High Energy Spectra
}

\author{
K. Tomita ${ }^{1}$ and T. Mizoguchi ${ }^{1}$ \\ ${ }^{1}$ Institute of Industrial Science, University of Tokyo, Japan
}

ELNES originates from electron transition from core orbital to conduction band, and the electron transition follows electron dipole transition. Thus, ELNES profile reflects the partial density of state of selected elements in the conduction band. By combining the aberration corrected STEM, electronic structure analysis with atomic resolution is possible. Thus, the EELS have been extensively observed for understanding the atomic and electronic structure of materials.

On the other hand, theoretical calculation is indispensable to interpret the ELNES. Together with the improvements of spatial and energy resolutions of experimental ELNES, the speed and accuracy of the theoretical calculation for ELNES are also improved. Recently, ELNES calculations using one-particle, two-particle (excitonic), and multi-particle methods have been systematically performed $^{[1]}$, and it was demonstrated that multi-particle theory is mandatory to calculate $\mathrm{L}_{2,3}$ edge of transition metals and $\mathrm{M}_{4,5}$ edge of lanthanides ${ }^{[2]}$, whereas two-particle (excitonic) theory is needed to reproduce ELNES of light elements, such as Li and Be. And, one particle calculation, which is conventional DFT-LDA/GGA method, can be applicable to ELNES except for those two cases ${ }^{\text {[3] }}$. However, importance of the excitonic effect on the high energy ELNES has not been discussed so far. In this study, we systematically performed the excitonic calculation, and the excitonic effects on both low and high energy ELNES were investigated. Furthermore, an application of the excitonic ELNES calculation to Li-ion battery was performed.

The ELNES calculation was performed using first principles Full-potential Linearized Augmented Plane-Wave (FLAPW) method based on DFT-GGA theory. The excitonic effect was included by solving the equation of motion for the electron-hole two-particle Green function, which is Bethe Salpeter Equation (BSE) ${ }^{[4]}$. As reported before, BSE makes better reproduction of ELNES for Li-Kedge of $\mathrm{LiCl}^{[1]}$ and $\mathrm{Al}-\mathrm{L}_{23}$ edge of $\mathrm{AlN}^{[2]}$ comparing with GGA. In this study, the excitonic calculations were performed for the low energy ELNES (less than $100 \mathrm{eV}$ ), such as $\mathrm{Na}-\mathrm{L}_{23}$ edge, $\mathrm{Mg}$ $\mathrm{L}_{23}$ edge and $\mathrm{Si}-\mathrm{L}_{23}$ edge, and the high energy ELNES (higher than 500eV), such as N-K edge, O-K edge, and Mg-Kedge. Furthermore, an application of the excitonic calculation for Li-ion battery was also performed.

Figure 1 shows the calculated and experimental $\mathrm{Na}-\mathrm{L}_{2,3}$ edge of $\mathrm{NaBr}$. All spectra were aligned using peak $C$. In the experimental spectrum, the sharp peaks $A_{1}$ and $A_{2}$ appear at the threshold and broad peaks $\mathrm{B}$ and $\mathrm{C}$ are followed. In the supercell calculation, although overall features can be reproduced, the position of the peak A is overestimated. On the other hand, the position of peak A becomes 
reproducible using the excitonic calculation. Similar trend has also reported in $\mathrm{Li}-\mathrm{K}$ edge of $\mathrm{Li}$ halides. However, it is found that the peak split at the threshold cannot be reproduced even in the excitonic calculation. We then tried BSE calculation with spin-orbit coupling. Peak splitting is observed and also relative peak intensity and position are well reproduced using the spin-orbit excitonic calculation. On the other hand, we found that the excitonic calculation and supercell calculation give the almost identical spectrum to each other in the case of MgO O-K edge, as shown in Fig.2.

We have systematically performed the excitonic calculation of low energy and high energy ELNES, and found that the excitonic effect is sometimes important even in the high energy ELNES. We are going to talk about those results in my presentation. Furthermore, some application of the excitonic calculation to the Li-ion battery will also be shown.

\section{References}

[1] T. Mizoguchi et al., Micron 41(2010) 695-709

[2] H. Ikeno et al., Phys. Rev. B, 83,155107 (2011)

[3] W. Olovsson et al., Phys. Rev. B, 79,041102 (2009)

[4] G. Onida et al., Rev. of Mod. Phys., Vol.74, Apr., 2002

[5] S. Nakai et al., Journal of the Physical Society of Japan, Vol.26, No.6, June, 1969

[6] T. Mizoguchi et al., Ultramicroscopy, 106(2006), 11201128

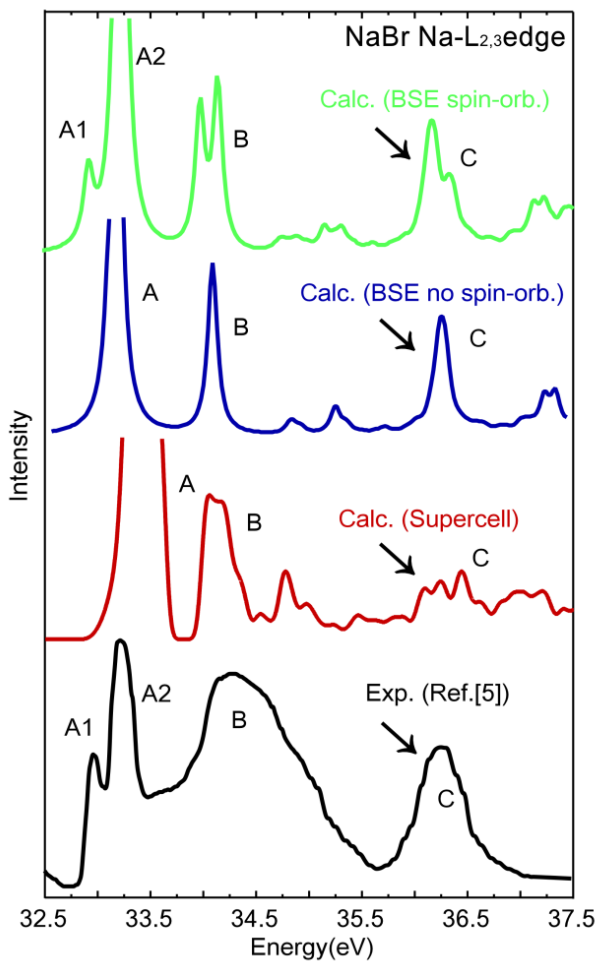

Fig.1 Experimental and calculated spectrum of $\mathrm{NaBr} \mathrm{Na}-\mathrm{L}_{2,3}$ edge

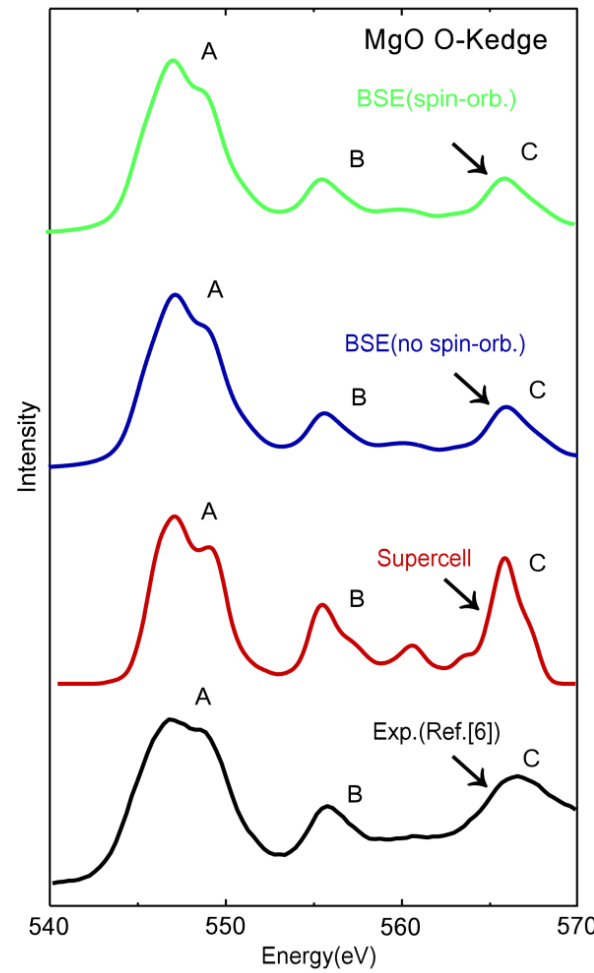

Fig.2 Experimental and calculated spectrum of $\mathrm{MgO}$ O-Kedge 